\title{
Relación del cambio climático con la producción agrícola en la Provincia del Azuay
}

\section{Relationship between climate change and agricultural production in the Province of Azuay}

David Zhindon-Pacheco

Universidad Técnica Particular de Loja, Ecuador

Priscilla Massa-Sánchez

Universidad Técnica Particular de Loja, Ecuador

Jesús Antonio Bonilla De Gracia

Universidad Técnica Particular de Loja, Ecuador

Autor para correspondencia: ezpintuclean@hotmail.com, pmassa@utpl.edu.ec, jabonilla2@utpl.edu.ec

Fecha de recepción: 01 de Junio de 2017 - Fecha de aceptación: 15 de Agosto de 2017

\section{Resumen}

La provincia del Azuay presenta tradicionalmente una concentración en los cultivos como el maíz, fréjol seco y tierno, arveja y papa, que además de constituir una actividad económica, son parte fundamental de la dieta, tradiciones y costumbres de la población. A partir de la sistematización de la información, se analiza la correlación que existe entre las variables, las características propias del cultivo y su relación con las variables climáticas. Los resultados alcanzados en el trabajo evidencian una relación negativa entre las variables analizadas; es decir, las variaciones en temperatura y precipitación disminuyen el rendimiento de los cultivos.

Palabras clave: temperatura; precipitación; productos primarios

\begin{abstract}
The Azuay province, traditionally has a concentration on crops such as corn, beans, peas and potatoes, which in addition to being an economic activity, are a fundamental part of the diet, traditions and customs of the population. From the systematization of the information, the correlation between the variables, the characteristics of the crop and its relationship with climatic variables is analyzed. The results obtained in the study show a negative relation between the analyzed variables. Variations in temperature and precipitation decrease crop yields.
\end{abstract}

Key words: temperature; precipitation; production; primary production 


\section{Introducción}

Del análisis de la vulnerabilidad de las actividades productivas ante variaciones climáticas, se deriva una amplia gama de relaciones que condicionan a la población concentrada en estas. Como hipótesis de investigación para evidenciar la existencia de una relación entre las variables climáticas y la actividad productiva se plantea realizar el análisis del nivel de correlación entre las variables productivas y asociadas al clima (temperatura y precipitación).

El interés en la investigación del tema remonta en la amplia evidencia empírica disponible y la experiencia de otras zonas en investigaciones alineadas. De acuerdo a la Fundación Carolina CeALCI (2012), en el informe referente al Impacto del cambio climático en la agricultura de subsistencia en el Ecuador, se prevé que los fenómenos de fuertes precipitaciones, olas de calor, sequías pueden llegar a representar para el sector agrícola erosión, saturación hídrica, pérdida de variedades, entre otros elementos que condicionan la continuidad rentable de la actividad agrícola.

Tomando como punto de partida la evidencia empírica se plantea contrastar la hipótesis para la provincia del Azuay e identificar posibles políticas que permitan de una manera adaptativa mitigar los posibles impactos o evitarlos.

La provincia del Azuay particularmente mantiene una tradicional concentración en ciertos cultivos, que a más de representar en algunos casos la principal actividad económica, es una actividad cultural y representativa de las costumbres ancestrales de su gente.

Es visible con el pasar de los años que la reorientación de la actividad agrícola en la provincia ha presentado una desconcentración de la actividad, sin embargo, es importante la participación de predios con un destino de autoconsumo en el que se mantienen cultivos tradicionales.

Los cultivos representativos a ser analizados en el estudio son: maíz, fréjol seco y tierno, arveja y papa. Para contrastar la hipótesis planteada en la investigación, la metodología aplicada es el análisis de las correlaciones entre las variables identificadas en los párrafos anteriores, que otorguen evidencia estadística para conducir a conclusiones congruentes con las investigaciones y estudios que existen en el área.

\section{La agricultura en el Ecuador y en la Provincia del Azuay}

La agricultura para el Ecuador ha representado históricamente una actividad económica relevante, un elemento determinante y característico de la concentración a nivel nacional en la actividad es el factor climático determinado por la ubicación geográfica, la variedad de temperaturas y precipitaciones, representa para el país una ventaja competitiva en la actividad.

El Ecuador por su caracterización en términos de variedad climática tiene una amplia diversidad de producción. El país se encuentra entre los cinco países con mayor diversidad biológica (Jiménez Sandra, Castro Luis, Yépez Javier \& Cristina Wittmer, 2012).

La distribución agrícola y su paisaje se encuentra distribuido entre las regiones Sierra, Costa y Amazonía (García, 2005). La actividad agro productiva en Ecuador se caracteriza por ser 
intensiva en mano de obra, el proceso de tecnificación, el camino a la transición de una agricultura tradicional a una denominada "agricultura no tradicional" refleja una alta intervención del estado bajo la aplicación de políticas e incentivos para el desarrollo de cultivos para exportación. De acuerdo a Damiani (2000) la agricultura no tradicional de exportación se presentó durante los años 1990s, siendo los principales productos las flores, frutas, hortalizas y cultivos orgánicos. Siendo estos productos altamente demandados y los que caracterizan al Ecuador a nivel internacional. La demanda de esta producción se deriva de los países industrializados, y representa un fuerte vínculo comercial.

De acuerdo al papel analizado del sector público en el desarrollo de los cultivos no tradicionales Damiani (2000) identifica ciertos factores:

- Políticas macroeconómicas y sectoriales

- Infraestructura: en caminos, servicios básicos, riego

- Desarrollo tecnológico

- Crédito

- Capacitación

- Generación de empleos

Específicamente para la provincia del Azuay la actividad económica agrícola representa el 2.86\% de acuerdo al GAD Provincial. Los cultivos más destacados dentro de los trasitorios son el Maíz suave seco que de acuerdo con el III Censo Agrícola es del 31,3\%, seguido del fréjol seco con el 27,9\%. Los monocultivos más importantes de la provincia son el maíz suave "Choclo" y la papa siendo los cantones con mayor concentración en estos cultivos Paute, Cuenca, Santa Isabel, Pucará y Nabón.

Dentro de los cultivos permanentes el cantón representativo de la Provincia es Pucará con la producción de banano y cacao, un aspecto determinante de esta concentración es la temperatura y precipitación para la concentración de esta producción, además los cantones que también producen estos cultivos son Santa Isabel y Camilo Ponce Enríquez por la misma condición climática.

\section{Cambio Climático y la Agricultura}

La vulnerabilidad de las variaciones climáticas y sus posibles afecciones sobre la producción agrícola, ha recobrado importancia en los últimos años, direccionando a los encargados de la política a tomar medidas para mitigar los potenciales impactos.

Los grandes desafíos a los que se enfrenta deben considerar como base todas las implicaciones que están directa e indirectamente vinculadas con los cambios climáticos. De acuerdo a los resultados del Encuentro Regional realizado en la Sede Central del IICA, en Costa Rica (Julio 2014), el crecimiento de la población mundial duplicará la demanda de alimentos para el 2050, este escenario visto en un contex to integral y bajo un supuesto de continuidad del ritmo de degradación de los recursos naturales, es una situación que agrava el problema relacionado con las tierras, la deforestación, pérdida de biodiversidad y alteración de las fuentes hídricas. 
Entre algunos de los impactos citados y ya identificados en estudios vinculados con el cambio climático son:

- $\quad$ Alteraciones o variaciones en los rendimientos y productividad de los cultivos, dentro de este aspecto, el aumento o disminución exagerada de las temperaturas tiene su efecto directo sobre las cantidades producidas

- Disponibilidad de fuentes de agua, las alteraciones climáticas a su vez repercuten en la disponibilidad del recurso hídrico, y las alteraciones de las cuencas abastecedoras del recurso.

- $\quad$ Aparición de plagas, pestes, malas hierbas, la proliferación de pestes en la agricultura es un tema que puede ser monitoreado en circunstancias, sin embargo, demanda de costos adicionales que, a más de encarecer el producto, lo tornan en un producto recargado de sustancias químicas que no necesariamente califican y garantizan una alimentación saludable. También se pueden presentar casos en los que exista pérdida total o parcial de los cultivos.

- $\quad$ Aceleración en pérdida de fertilidad de los suelos, de acuerdo a los microambientes generados y la demanda del suelo para mantener su producción, se pueden presentar casos en los que los suelos tienden a perder su propiedad de restitución vegetativa, pasando a demandar de productos químicos que palean la situación a corto plazo, sin embargo, comprometen la actividad en un mediano y largo plazo.

El Ecuador particularmente como parte de su política y considerando su estructura productiva entorno al sector primario ha adoptado medidas que permitan regular y controlar el uso y manejo de los recursos hídricos, dispone de programas para el fomento productivo con implementación de tecnología, asesoramiento en el manejo de los cultivos y uso de agroquímicos. Todas estas medidas de una u otra forma representan acciones para contrarrestar y manejar los impactos, que simultáneamente permiten una estructura más sólida de la actividad económica.

\section{Metodología}

En base a la revisión de literatura y de casos de estudio tanto nacionales como internacionales y la evidencia existente entre las variables climáticas, específicamente entre la precipitación y temperatura, con respecto a la producción agrícola. De la presente investigación el objetivo es probar la existencia significativa de estas relaciones para la producción representativa de la provincia del Azuay, con su respectiva información de temperatura y precipitación durante el período 2010 - 2012. La metodología empleada para el análisis se basa en dos perspectivas, la primera se enfoca en realizar un análisis descriptivo histórico de las características del cultivo, su patrón de comportamiento (superficie sembrada, cosechada, producción ventas), las características de adaptación climática del cultivo, entre otros aspectos relevantes que describan al producto y permitan generar un marco completo. Como segundo enfoque, se desarrolla el análisis estadístico de correlación entre las variables, con la finalidad de probar la existencia de una relación entre las variables. El análisis estadístico se realiza obteniendo el Coeficiente de Correlación de Pearson (CCP) definido este como un estadístico que prorratea los niveles de covarianza entre las variables, sobre el producto de sus desviaciones típicas. 


$$
\rho_{X, Y}=\frac{\sigma_{X Y}}{\sigma_{X} \sigma_{Y}}=\frac{E\left[\left(X-\mu_{X}\right)\left(Y-\mu_{Y}\right)\right]}{\sigma_{X} \sigma_{Y}}
$$

Los resultados del CCP oscilan entre absolutos oscilan 0 y 1 . No obstante los resultados del coeficiente pueden variar dentro de este mismo rango a valores negativos. En este sentido una fuerte relación positiva está representada por valores lo más próximos a +1 , por otra parte, una fuerte relación negativa se presenta en valores lo más próximos a -1. Los resultados del límite, es decir $+1,-1$ se interpreta como una relación perfecta positiva y perfecta negativa. En los siguientes acápites se realiza los análisis para identificar de manera descriptiva y estadística la relación entre las variables climáticas (temperatura y precipitación), con las variables de producción (rendimiento, TM producidas).

\section{Resultados}

\section{Resultado del Análisis de correlación entre precipitación, temperatura y producción en la Provincia del Azuay}

Tabla 1. Relación bivaridad entre producción y precipitación.

\begin{tabular}{cccc}
\hline \multicolumn{4}{c}{ Producción } \\
\hline Precipitación & Arveja & $\begin{array}{c}\text { Frejol } \\
\text { seco }\end{array}$ & $\begin{array}{c}\text { Frejol } \\
\text { tierno }\end{array}$ \\
& -0.55 & -0.37 & -0.31 \\
\multicolumn{4}{c}{ Producción } \\
Precipitación & Maíz & Papa \\
& -0.60 & -0.45 &
\end{tabular}

Nota. Fuente: Zhindón, D., Massa, P. y Bonilla. (2017).

En la tabla 1 se muestran los resultados obtenidos entre la relación bivariada entre producción y precipitación. De los resultados obtenidos se puede concluir que presentan una consistencia en el signo de la correlación, y el grado de correlación varía entre débil a fuerte en el caso del maíz que presenta el mayor coeficiente. Estos resultados se corroboran analizando los niveles adaptativos de la producción con la información disponible y su variabilidad.

Tabla 2. Relación bivaridad entre producción y precipitación.

\begin{tabular}{cccc}
\hline \multicolumn{4}{c}{ Rendimiento } \\
\hline Temperatura & Arveja & $\begin{array}{c}\text { Frejol } \\
\text { seco }\end{array}$ & $\begin{array}{c}\text { Frejol } \\
\text { tierno }\end{array}$ \\
& -0.41 & -0.70 & -0.43 \\
Rendimiento & \\
Temperatura & Maíz & Papa \\
& -0.47 & -0.42 &
\end{tabular}

Nota. Fuente: Zhindón, D., Massa, P. y Bonilla. (2017).

En la tabla 2 se muestra la correlación con la temperatura se obtuvieron los siguientes resultados. Los signos de los coeficientes evidencian de igual manera una relación negativa, siendo el fréjol seco el de mayor grado de correlación negativa con respecto a la variación de la 
temperatura. Los demás productos evidencian una correlación que bordea el nivel medio, reflejando su vinculación con la variabilidad climática. La dispersión de los datos observados para el período de análisis 2000 -2012 de los productos y la correlación lineal que se presentan entre los datos se detallan en las siguientes imágenes de los resultados obtenidos:

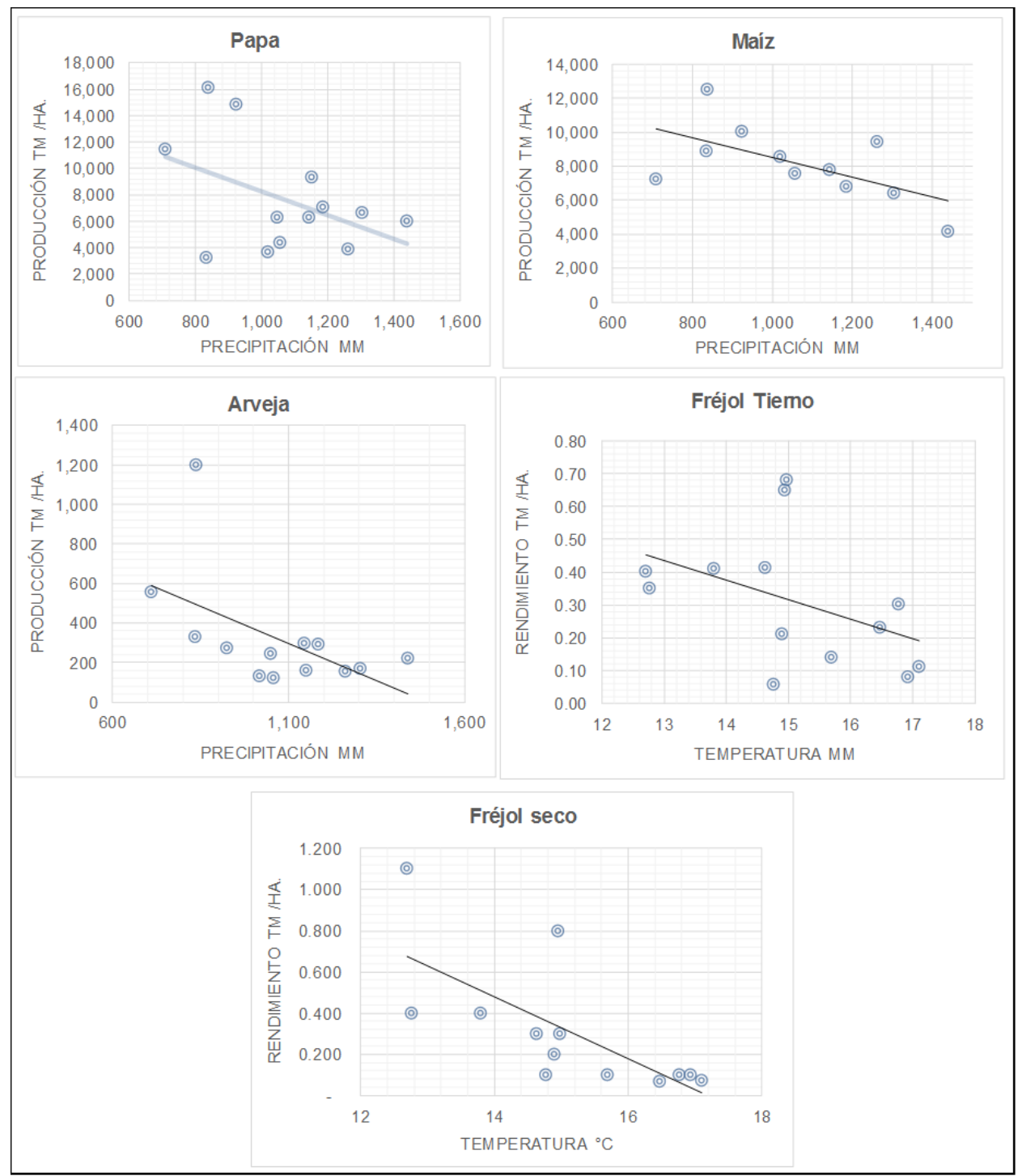

Figura 1: Dispersión rendimiento, producción -precipitación, temperatura. Fuente: (Instituto Interamerciano de Cooperación para la Agricultura, 2014). Elaborado por: Los autores.

\section{Recomendaciones de Política Pública}

El agro para el Ecuador representa un pilar importante en términos de su participación en el PIB, por lo que entre otros los riesgos que se identifican dentro de la actividad son: la migración del campo a la ciudad, el desincentivo en la concentración en la actividad, la legislación vinculante a la tenencia de tierras, las limitaciones de crédito y técnicas de la población concentrada en la actividad, los canales de comercialización no totalmente desarrollados, entre otros aspectos legales que fortalezcan la actividad y permitan garantizar su rentabilidad (Uribe, 2014) 
Entre otras instituciones, el MAGAP como actor rector en los temas productivos, ha impulsado ciertas políticas para fomentar e incentivar la actividad mediante programas que buscan la optimización de recursos, tecnificación de procesos, reducción de costos, esto fue abordado en el Acuerdo Ministerial 524 MAGAP, firmado el 7 de noviembre de 2013.

Entre las disposiciones más relevantes del Acuerdo Ministerial se pueden enumerar:

- Disponer de la Empresa Pública Unidad Nacional de Almacenamiento "UNA EP"

- Reducir el margen de intermediación en el precio del aceite agrícola, en base de un convenio con la Empresa Pública Petroecuador. Complementariamente, facilitar el trámite de aprobación de la obtención de aceite agrícola y diésel por parte de asociaciones del sector.

- Realizar a través de la Unidad competente del Ministerio, el análisis de suelos y aguas sin costo para el productor

- Revisar los costos de exportación en cartón, plásticos y gastos aeroportuarios

- Operativizar los procesos para que el IESS otorgue a favor de los productores un período de amnistía que permita regular la afiliación de los trabajadores agrícolas

- Solicitar al IESS procesos de entrada y salida de afiliación de los trabajadores agrícolas más flexibles

Desde varios enfoques las políticas públicas deben considerara y afrontar la problemática relacionada con la vulnerabilidad del cambio climático y la actividad agrícola, ya que entre las potenciales consecuencias la desconcentración en esta actividad económica puede generar un desequilibrio entre la oferta y demanda de los productos.

Entre los varios aspectos que deben ser considerados por las políticas públicas, estas deberían considerar:

- Monitoreo de los niveles de producción y sus concentraciones, así como las causas de abandono de la actividad.

- Registro fiable de información de superficie y rendimientos de la producción.

- Seguimiento al estado de conservación de recursos del territorio como, fuentes hídricas de abastecimiento para riego, y necesidades de la población de acuerdo a los requerimientos de producción.

- Realizar proyectos para de manera planificada y coordinada con los involucrados directos, tomar medias que mitiguen los impactos como: reservorios de agua para riego, capacitación continua para la adaptación de cultivos y posibles amenazas que se presenten.

- Particularmente la provincia del Azuay cuenta con reservas boscosas amplias que son la base para el mantenimiento de las fuentes hídricas, debiendo ponerse énfasis en su cuidado y protección

- Medidas de protección financiera para los productores, a través de la disponibilidad de seguros que cubran los seguros, sobre todo aquellos que demandan de una alta inversión dentro de su proceso productivo

- Realizar la planificación territorial, en el que se definan la frontera agrícola y la definición de áreas mínimas de producción generando incentivos en la concentración de ciertas actividades y des incentivando la concentración de cultivos no productivos. Esto sería a través de realizar programas de uso eficiente del suelo. 
- Identificar y generar bolsas de recursos que permitan afrontar presentes y potenciales impactos asociados a las variaciones climáticas, para apalancar a los productores y mantener la concentración en la actividad.

A nivel específico de la zona analizada, es importante considerar programas que incentiven la concentración en la actividad productiva primaria, y esto no represente un abandono del sector primario productor. La problemática debe considerarse para evitar potencializarla y que presente un trasfondo en la vulnerabilidad económica del sector agro productivo.

\section{Conclusiones}

De los resultados obtenidos a través del coeficiente de correlación y la recopilación de la evidencia empírica. Para la provincia del Azuay se corrobora la hipótesis planteada, es decir existe una vulnerabilidad en términos de producción agrícola vinculado a la variación climática (temperatura y precipitación).

Los cultivos representativos analizados de la provincia del Azuay y su nivel de correlación con las variaciones climáticas y de precipitación presentan una relación negativa para todos los casos analizados (Arveja, Fréjol seco, Fréjol Tierno, Maíz y Papa). El signo de la correlación en primera instancia alerta sobre las repercusiones actuales y potenciales que se podrían derivar bajo un escenario de alta variabilidad de temperatura o precipitación. Los resultados de correlación entre los volúmenes de producción y precipitaciones varían entre -0.31 (Fréjol tierno) hasta -0.60 (Maíz), obtenidos estos resultados, se observa que una relación negativa entre débil y significativa, otorgando un indicio o concordando con la evidencia empírica.

Por parte de la correlación entre el rendimiento y temperatura, los resultados obtenidos varían entre -0.41 a -0.70 , siendo el Fréjol seco el que presenta la correlación más fuerte, y la Arveja con - 0.41 de coeficiente de correlación presenta la relación inversa menor. Estos resultados deben ser considerados como una base para continuar con monitoreos similares en el mediano y largo plazo que posibiliten dimensionar y con mayores análisis cuantificar las repercusiones económicas y financieras para los concentrados en la actividad agrícola.

La arveja, maíz y papa son los productos que presentan una mayor variabilidad con respecto a la precipitación, que con respecto a la temperatura, siendo el fréjol seco el que mayor diferencia presenta, estos comportamientos deben ser monitoreados para identificar un comportamiento y su medida de control. El maíz es un producto representativo y característico dentro de la dieta de la población del Azuay, del análisis de obtiene que mantiene una significativa correlación ante las variables climáticas, es decir estas condicionan los niveles de producción y rendimientos que se obtienen de este cultivo.

De los diferentes análisis e investigaciones se puede evidenciar que las variables ambientales y climáticas como la temperatura y precipitación condicionan ciertos factores de la actividad económica, sobre todo de la agrícola que está directamente vinculada con el medio ambiente. La temperatura y los demás factores condicionan entre otros elementos de los factores productivos los relacionados al suelo y su equilibrio en la composición de nutrientes que garantizan la continuidad de los rendimientos de los cultivos. La temperatura es un elemento importante al 
momento de analizar el proceso biológico de los suelos y sus variaciones o alteraciones, esto acorde a la investigación se corrobora para la provincia del Azuay.

Finalmente, a más de las implicaciones directas que pueden representar las variaciones climáticas negativamente sobre los niveles de rendimiento y consecuentemente en la sostenibilidad económica de la actividad productiva. Se derivan elementos indirectos que pueden alterar el equilibrio productivo demandando de mayores recursos económicos (por ejemplo: para contrarrestar plagas o alteraciones en las condiciones de los suelos) o humanos (mayor demanda de trabajo para controlar malezas), o hasta desplazando a la población económicamente activa concentrada en esta actividad hacia otra actividad que potencialmente represente mayor rentabilidad.

\section{Bibliografía}

Agosta, E. Cavagmarp, M. Panziani, P. (2010). El rendimiento de VID y las variaciones de temperatura y precipitación en Mendoza. Revista Ecológica.

Altieri, M. \& Nicholls, C. (2009). Cambio climático y agricultura campesina: impactos y respuestas adaptativas. LEISA.

Bollin, C. Komitee, D. (2011). Análisis sobre adaptación al cambio climático, un instrumento para la consideración del cambio climático y sus efectos en los programas y proyectos de Welthungerhilfe.

Carvajal, F. (2013). Cambio climático y costos de producción. OSPA Observatorio de Política Socio Ambiental - PUCE Pontificia Universidad Católica de Ecuador.

CEPAL. (2011). Agricultura y cambio climático: instituciones, políticas e innovación. Memoria del seminario internacional 2010. Santiago de Chile.

Fernández, M. (2013) Efectos del cambio climático en el rendimiento de tres cultivos mediante el uso del Modelo AquaCrop. Colombia.

Fernández, M. (2013) Efectos del cambio climático en la producción y rendimiento de cultivos por sectores. Colombia. FONADE

Food and Agriculture Organization (FAO). (2009) Adaptación de la agricultura al cambio climático.

Food and Agriculture Organization (FAO). (2013) Cambio Climático, El impacto en la agricultura y los costos de adaptación. Instituto Internacional de Investigación sobre Políticas Alimentarias, Washington, D.C.

Gobierno Provincial del Azuay. Plan de Desarrollo y Ordenamiento territorial del Azuay 2015-2030

Ibáñez, D. (2011). Efectos del cambio climático en las actividades agrarias y forestales. España. Universidad de Alicate

Iñiguez, K. (2015). Impacto del cambio climático en la producción de café, caso de estudio: Asociación "PROCAFEQ" - cantón Quilanga 2001 -2010. Ecuador. 
Jiménez, A. Massa, P. (2015). Producción de café y variedades climáticas. El caso de Espíndola. Ecuador.

Ludeña, C., Wilk, D. (2013). Ecuador: Mitigación y Adaptación al Cambio Climático, Marco de preparación de la Estrategia 2012 -2017 del BID en Ecuador

Marchán, J. (2009). Diálogos sobre el cambio climático - seguridad alimentaria. Quito

Martínez, L. (2004) Dinámicas rurales en el sub trópico. CAAP. Quito.

Mora, J. Ramírez, D. Ordaz, J. Acosta, A. Serna, B. (2010). Efectos del Cambio climático sobre la agricultura. Costa Rica.

Mora, J. Ramírez, D. Ordaz, J. Acosta, A. Serna, B. (2010). Efectos del Cambio climático sobre la agricultura. Panamá.

Muñoz, G. (2007) El reto de la agricultura frente al cambio climático. CEDA.

Noboa, S. Castro, L. Yépez, J. Wittmer C. (2012). Impacto del cambio climático en la agricultura de subsistencia en el Ecuador, Fundación Carolina CEALCI. Madrid

Ocampo, O. El cambio climático y su impacto en el agro.Revista de Ingeniería. DOSSIER.

Ordaz, J. Ramírez, D. Mora, J. Acosta, A. Serna, B. (2010). Efectos del Cambio climático sobre la agricultura. Guatemala.

Ordaz, J. Ramírez, D. Mora, J. Acosta, A. Serna, B. (2010). Efectos del Cambio climático sobre la agricultura. El Salvador.

Ortiz, R. (2012) El cambio climático y la producción Agrícola. Banco Interamericano de Desarrollo.

Peralta, E. Murillo, A. Mazón, N. Monar, C. Pinzón, J. Rivera, M. (2010). Manual agrícola de fréjol y otras leguminosas. Publicación Miscelánea No. 135. Quito.

Pinto, Máximo. (2007). El cultivo de arveja y el clima en el Ecuador. INAMHI.

Quiroga, S. Iglesias, A. (2000). Influencia del clima sobre la productividad agrícola en España. Madrid.

Ramírez, D. Ordaz, J. Mora, J. Acosta, A. (2010). Efectos del cambio climático sobre la agricultura. CEPAL. Nicaragua

Torres, L. (2010). Análisis económico del cambio climático en la agricultura de la región Piura - Perú. Caso: principales productos agroexportadores.

Vergara, W. Rios, A. Trapido, P. Malarín, H. (2014). Agricultura y Clima Futuro en Amética Latina y el Caribe: Impactos Sistémicos y posibles respuestas. Banco Interamericano de Desarrollo. 